\title{
What is a crystal: The question comes full circle
}

\author{
Larry R. Falvello \\ University of Zaragoza - CSIC, Department of Inorganic Chemistry and Aragón \\ Materials Science Institute (ICMA), Pedro Cerbuna 12, 50009 Zaragoza, Spain. \\ falvello@unizar.es
}

With reference to personal experiences with crystals, a reflection is presented on the nature of crystals, their structures and their transformations. Two "standard" definitions and one official definition of a crystal are offered as a point of departure for a discussion of the ways in which crystals are used in chemistry and of some possible practical variants on the definition of crystals. The key properties of periodicity and symmetry, and the nature of motions within crystals, are used in further development of an overview of their defining characteristics. It is concluded that a context-dependent, functional or phenomenological definition is as useful in practical applications as is a rigorous firstprinciples definition. 